\title{
Imprecision and bias in orthodontic treatment results
}

\author{
Michael L. Rlolo, D.D.S., M.S.," Robert E. Moyers, D.D.S., Ph.D.,"* and \\ Thomas R. TenHave, M.P.H.*** \\ Ann Arbor, Mich.
}

Imprecision in treatment response has been defined as inconsistent unpredictable results from the same treatment. Bias has been defined as systematic failure to achieve defined treatment goals. Concepts of imprecision and bias are applied to the results of a study of soft-tissue response to Class II treatment with edgewise and Herbst appliances. (AM J ORTHOD DENTOFAC ORTHOP 1988;93: 138-42.)

$\mathrm{O}_{\mathrm{r}}$ rthodontists are interested in the reasons some cases respond to treatments better than others, but it is difficult to assign the responsibility for "success" or "failure" among such varied factors as skeletal morphology, patient compliance, skill of the orthodontist, maturational age of the patient, and efficiency of the appliance. Furthermore, some goals of treatment are difficult to quantify despite the routine use of cephalometrics, for example, improved function and more pleasing facial esthetics.

In recent years the methods of clinical research in medicine have become much more sophisticated largely through analytic procedures developed by research teams (Feinstein ${ }^{1}$ and Sackett, Hayes, and Tugwell ${ }^{2}$ ) and a new field termed clinical epidemiology has emerged. Orthodontics is well suited to profit from these new strategies for clinical research, but we have been reluctant to abandon the traditional anecdotally oriented clinical report for the more practical modern approaches pioneered by Sackett's and Feinstein's groups.

It may be more difficult to quantify esthetic goals of orthodontic intervention and we have not yet been able to segregate the continuing growth from treatment response cleanly and routinely, but some commonly accepted goals of treatment are measurable and thus should be emphasized in studies of treatment effects. This article defines and demonstrates two concepts involved in the study of treatment effects-imprecision and bias - and shows how their understanding and use in clinical studies would lead to improved appreciation of orthodontic treatments.

From the Center for Human Growth and Development, University of Michigan. *Research Scientist.

**Fellow and Professor of Dentistry.

***Research Associate.

\section{DEFINITIONS}

How do clinicians fail to achieve treatment goals? Fig. 1 illustrates two possible ways. Part $A$ depicts a well-defined goal that seems difficult to achieve since a consistent shortfall of the expected goal in treatment response is produced. Thus, a bias in treatment response is revealed. ${ }^{3}$ The mean response to this particular treatment, as well as all individual responses, falls short of the planned treatment goal, but the range and variation in treatment response are small.

Part $B$ of Fig. 1 illustrates a well-defined goal that is seemingly achievable on the basis of mean treatment response. However, in this case it is difficult to predict any individual treatment response since the range of responses is very great, though centered around the goal. Instead of systematic bias, there is shown a large random error-imprecision-that gives an impression of predictable response.

Fig. 2 illustrates an alternative representation of the same data to contrast further bias with imprecision. The dot in the center of the circle in $A$ represents the goal; the large lower dot depicts the mean treatment response around which the individual responses are tightly clustered-well-defined bias.

In $B$ of Fig. 2, the dot indicates that the mean treatment response is congruent with the goal of the treatment. However, the spread of the individual responses around their mean is great. Because of this imprecision, an individual case may or may not actually achieve the planned goal in spite of the absence of bias in mean response. The tightly clustered shortfall is easier to predict; the wide-ranging scatter is far less precise even though the mean response is exactly coincident with the planned goal.

How might one compensate for inability to achieve goals? Systematic bias clearly suggests setting a more 
How do we miss our goals?

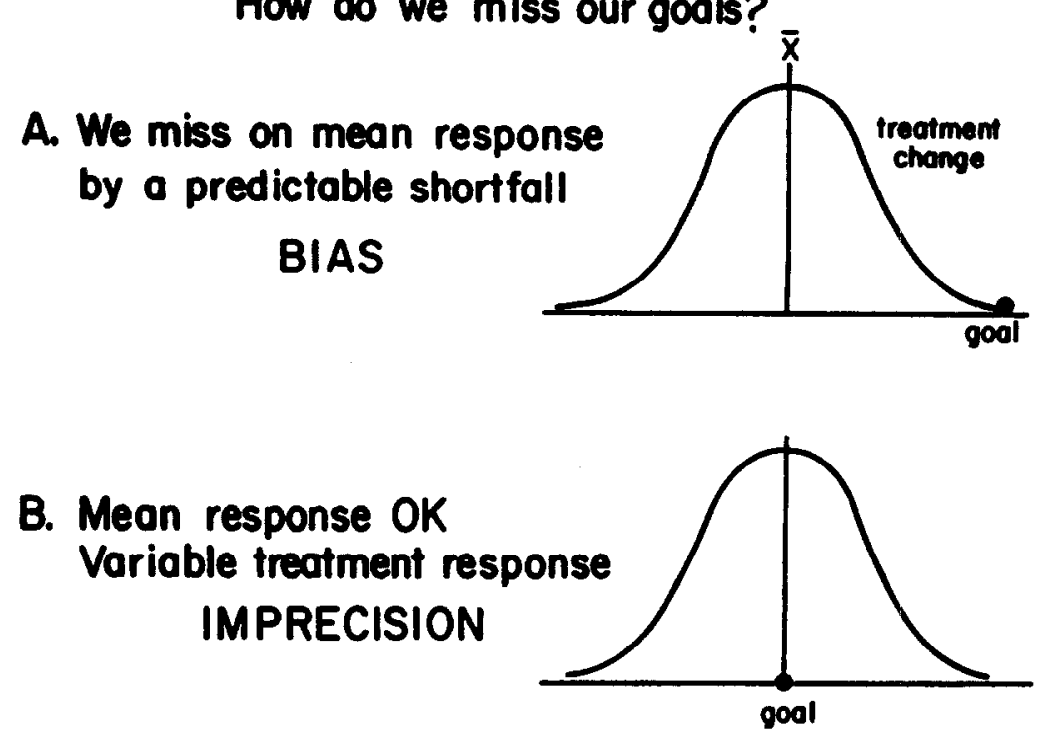

Fig. 1. Graphic comparison of bias and imprecision.

\section{How do we compensate for our inability to achieve our goal?}

\section{GOAL}

A. BIAS
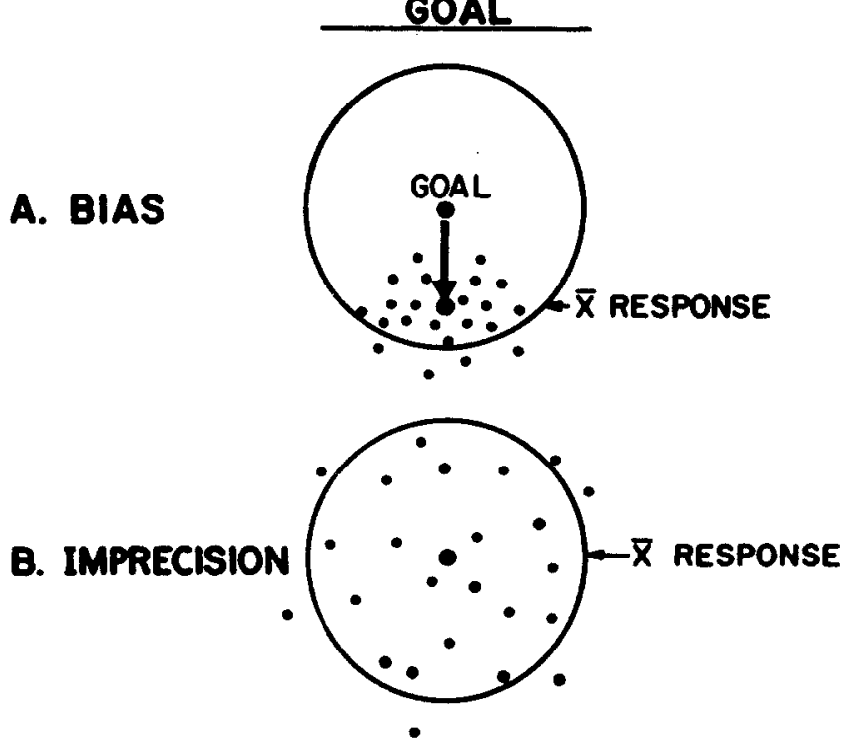

Fig. 2. Another depiction of bias and imprecision.

realistic goal or modifying the treatment to achieve the objective. Imprecision may be reduced by better patient selection, more consistent treatment planning, or an improved understanding of the mode of appliance action.

\section{APPLICATION OF CONCEPTS OF BIAS AND IMPRECISION \\ Source of data}

To illustrate the practical application of bias and imprecision in clinical reports, data from a study of the 


\section{PLOT OF ANNUAL CHANGE AT SPOG FOR HERBST (०) AND NON-EXTRACTION EDGEWISE (•) FEMALES}

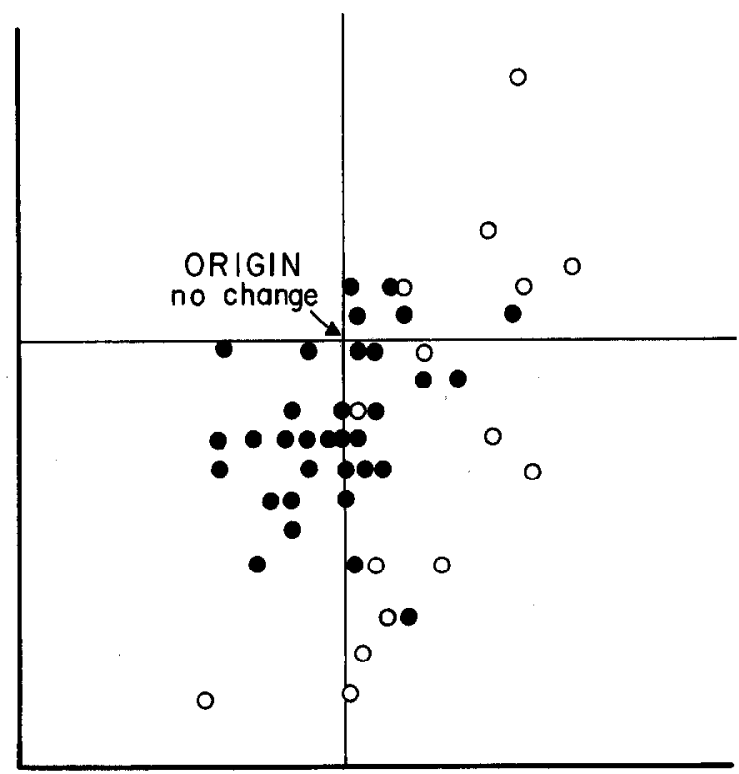

Fig. 3.

effects of two different appliances on the soft-tissue profile in Class II treatments were chosen. Compared here in Figs. 3 and 4 are the graphic results of the Herbst appliance $(\mathrm{N}=43)(\mathrm{McNamara}$ and associates, study in preparation) and edgewise nonextraction cases $(\mathrm{N}=70)^{4,5}$

For the purposes of this article, it is sufficient to display graphically the response of but one soft-tissue landmark (soft-tissue pogonion) to demonstrate the clinically important differences between bias and imprecision.

Variance (square of standard deviation) and range give estimates of imprecision, but estimates of bias cannot be presented because the planned treatment changes were not quantified. If treatment goals for each individual had been specified, bias, as defined earlier herein, could have been estimated. Quantified estimates of bias cannot be shown without quantified expectations.

\section{Examples}

Clear graphic examples of bias and imprecision are found in the data from our study of soft-tissue profile response to different orthodontic appliances used in Class II therapy. Fig. 3 is a plot of annual changes in the position of soft pogonion relative to sella-nasion with sella as the origin. The filled and open circles represent edgewise nonextraction and Herbst appliance cases, respectively, for a group of female patients. The intersection in the center represents no change on either the $\mathrm{x}$ or $\mathrm{y}$ axis and all the circles are the posttreatment positions relative to the pretreatment positions. Note that the edgewise responses tend to cluster near the center of the plot, while the Herbst responses are more widespread above, below, and to the right of the edgewise cases.

All the expected goals typically would be somewhere to the right of the center of this diagram yet the edgewise female patients unfortunately fall systematically below and to the left of the goal. On the other hand, the Herbst female patients are distributed to the right of the zero response point (origin), exhibiting more variation than the edgewise female patients. Changes resulting from untreated growth should tend to array themselves downward and forward (right on the plot) from the intersection.

The responses of male patients are shown in Fig. 4. Although sex differences in treatment response are not the topic of this report, it is interesting to note the randomness of the responses to Herbst therapy in both sexes (imprecision) and the systematically poorer response of Class II female patients to edgewise treatment. Table I demonstrates the greater imprecision of 


\section{PLOT OF ANNUAL CHANGE AT SPOG \\ FOR HERBST(0) AND NON-EXTRACTION EDGEWISE (•) MALES}

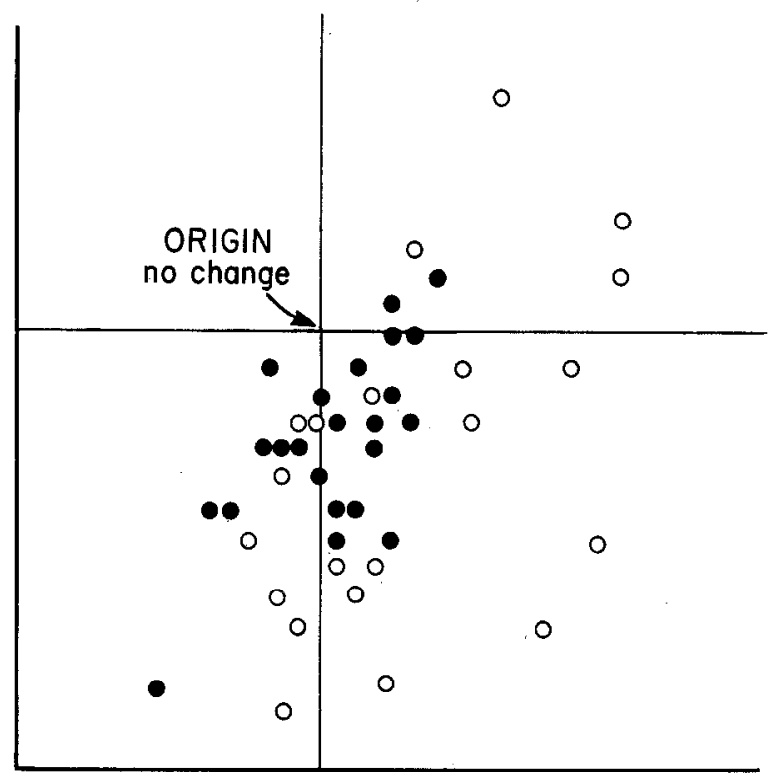

Fig. 4.

the Herbst treatment. The apparent bias among female patients in the Edgewise sample is poorer from a clinical view (pogonion is more inferior and dorsal), but more tightly clustered, thus more predictable than that in the male patients with the same treatment.

\section{DISCUSSION}

Some of the variability present in these results is caused by the retrospective aspect of the study. Many other unknowns, such as the varying skills of the individual orthodontists, the details of each treatment plan, and the different goals, may obscure possible differences in treatment effects. Additional characteristics of this and other studies that may obfuscate our understanding of the effects of treatment include:

1. The lack of a comparable untreated control sample, especially important in view of varying Class II morphologies

2. The heterogeneity of the treatment samples with respect to treatment interval, age, pretreatment skeletal and soft-tissue morphologies, and patient compliance

3. The use of measures of central tendency when variability of treatment response is so great

However, it seems quite clear that where treatment goals are well defined, as in desired ventral changes of soft-tissue pogonion in Class II treatments, it is possible
Table I. Measures of bias and imprecision of annual change in the sella-nasion-soft pogonion tensor

\begin{tabular}{c|c|c}
\hline $\begin{array}{c}\text { Treatment } \\
\text { group }\end{array}$ & Imprecision & Bias \\
\hline $\begin{array}{c}\text { Male patients } \\
\text { Herbst }\end{array}$ & 7.0 & \\
Edgewise & 2.4 & $?$ \\
Female patients & & $?$ \\
Herbst & 4.5 & $?$ \\
Edgewise & 2.3 & $?$ \\
\hline
\end{tabular}

'Imprecision is computed as 1000 times the square root of the sum of the variances of the annual changes in the $\mathrm{x}$ and $\mathrm{y}$ coordinates of the sella-nasion-pogonion tensor.

to derive improved understanding of treatment effects through study of both bias and imprecision. Other landmarks, other goals, and different morphologies are easily available variations on this theme.

The clinician is more concerned with individual treatment effect than mean treatment effect with which bias and imprecision are formulated. Therefore, bias and imprecision may be more acceptable when treated together as root-mean-square error, ${ }^{3}$ the error between observed individual treatment effects, and the expected 


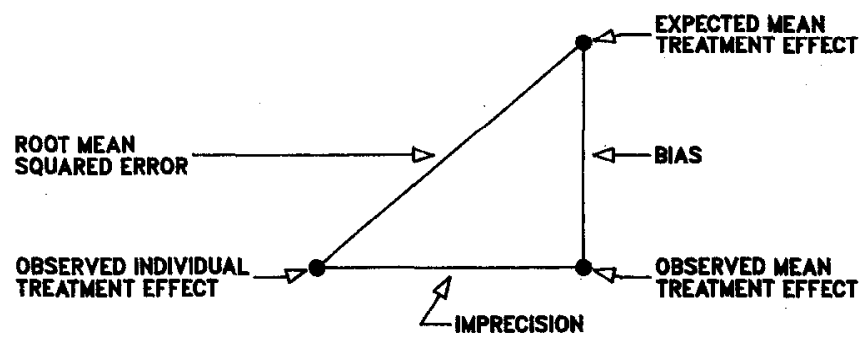

Fig. 5. A, Diagram illustrating the geometry of the mathematic relationship among bias, imprecision, and root-mean-squared error.

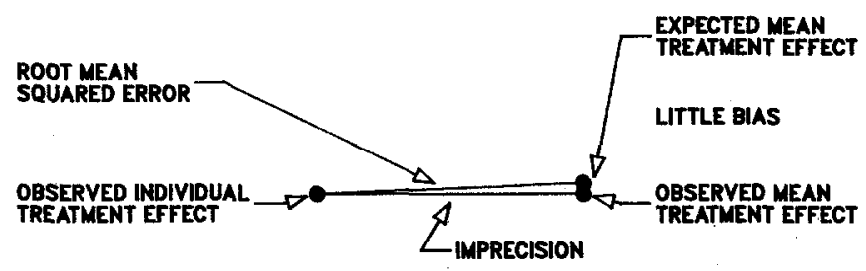

Fig. 5 (Cont'd). B, Diagram depicting the relationship when there is little bias relative to imprecision.

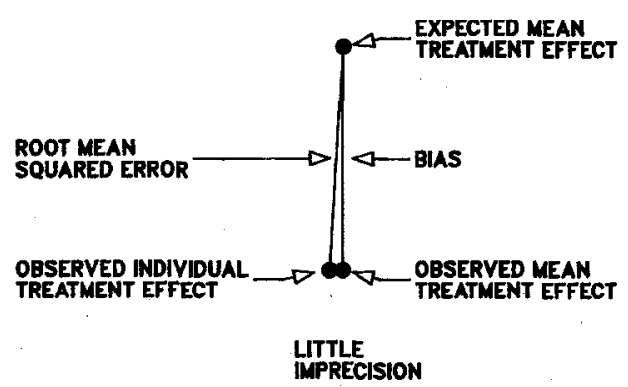

Fig. 5 (Cont'd). C, Diagram demonstrating greater bias relative to imprecision. This figure is based on a mathematic model and therefore does not correspond to the plots of treatment change in Figs. 3 and 4.

trcatment effect (or the treatment goal conceived before treatment, see Fig. 5, A). To minimize effectively the root-mean-square error, both bias and imprecision must be quantified and controlled. Reducing one while inflating the other will likely yield individual treatment effects that deviate significantly from the planned or expected effect (see Fig. 5, $B$ and $C$ ). Both bias and imprecision should be minimized so that the clinician can achieve the desired treatment effect more predictably.
We wish to thank Dr. James McNamara for the Herbst treatment data.

\section{REFERENCES}

1. Feinstein AR. Clinical biostatistics. St. Louis: The CV Mosby Company, 1977.

2. Sackett DL, Hayes RB, Tugwell P. Clinical epidemiology: A basic science for clinical medicine. Boston: Little, Brown \& Company, Publishers, 1985.

3. Deming WE. Some theory of sampling. New York: J Wiley \& Sons, 1950.

4. Riolo ML, TenHave TR. The effect of different kinds of appliance therapy on the facial soft-tissue profile. In: Vig PS, Ribbens KA, eds. Science and clinical judgment in orthodontics. Monograph 19, Craniofacial Growth Series. Ann Arbor: 1986. Center for Human Growth and Development, University of Michigan.

5. Riolo ML, Moyers RE, TenHave TR, Mayers MA. Facial softtissue changes during adolescence. In: Carlson DS, Rihbens KA, eds. Craniofacial growth during adolescence. Monograph 20, Craniofacial Growth Series. Ann Arbor: 1987. Center for Human Growth and Developmeni, University of Michigan.

Reprint requests to:

Dr. Michael L. Riolo

Center for Human Growth

$300 \mathrm{~N}$. Ingalls

University of Michigan

Ann Arbor, MI 48109 